\title{
Role of Working Memory Storage and Attention Focus Switching in Children's Comprehension of Spoken Object Relative Sentences
}

\author{
Mianisha C. Finney, ${ }^{1}$ James W. Montgomery, ${ }^{1}$ Ronald B. Gillam, ${ }^{2}$ and Julia L. Evans ${ }^{3}$ \\ ${ }^{1}$ Communication Sciences and Disorders, Ohio University, Athens, OH 45701, USA \\ ${ }^{2}$ Communication Disorders and Deaf Education, Utah State University, Logan, UT 84322, USA \\ ${ }^{3}$ Communication Sciences and Disorders, University of Texas at Dallas, Richardson, TX 75080, USA \\ Correspondence should be addressed to James W. Montgomery; montgoj1@ohio.edu
}

Received 2 March 2014; Revised 30 April 2014; Accepted 30 April 2014; Published 20 May 2014

Academic Editor: Nobuo Masataka

Copyright ( 2014 Mianisha C. Finney et al. This is an open access article distributed under the Creative Commons Attribution License, which permits unrestricted use, distribution, and reproduction in any medium, provided the original work is properly cited.

\begin{abstract}
The present study evaluated a two-mechanism memory model of the online auditory comprehension of object relative (OR) sentences in 7-11-year-old typically developing children. Mechanisms of interest included working memory storage (WMS) and attention focus switching. We predicted that both mechanisms would be important for comprehension. Forty-four children completed a listening span task indexing WMS, an auditory attention focus switching task, and an agent selection task indexing spoken sentence comprehension. Regression analyses indicated that WMS and attention focus switching accuracy each accounted for significant and unique variance in the children's OR comprehension after accounting for age. Results were interpreted to suggest that WMS is important for OR comprehension by supporting children's ability to retain both noun phrase 1 and noun phrase 2 prior to their reactivating noun phrase 1 from memory in order to integrate it into a developing structure. Attention focus switching was interpreted to be critical in supporting children's noun phrase 1 reactivation, as they needed to switch their focus of attention momentarily away from ongoing language processing to memory retrieval.
\end{abstract}

\section{Introduction}

Children find object relative (OR) structures difficult to process and understand across many languages, including English [1, 2], Hebrew [3, 4], Hungarian [5], and Italian [6, 7]. The difficulty with ORs (The lion that the monkey had bitten on the nose ran into the jungle) from a syntactic perspective can be attributed to their greater complexity. In English, one index of complexity relates to word order, with OR sentences violating typical or canonical subject-verb-object (SVO) word order. Noun phrase 1 (NP1) occupies the subject position but functions grammatically as the object and semantically as the patient. To recover an SVO structure from an OR sentence and understand "who did what to whom," the comprehender must perform a movement operation whereby NP1 "moves" from its fronted object position to its canonical postverb position (the monkey had bitten the lion).
Object relatives are difficult also because presumably they impose greater working memory (WM) demands. Minimally, the comprehender must be able to hold both unintegrated NPs in memory until hearing the embedded VP. Upon processing the VP, the comprehender then must reactivate NP1 from memory to "move" it to its postverb slot. Childhood studies directly focusing on the association between memory and online comprehension of OR sentences are surprisingly scarce.

The purpose of the present study was to begin to address this gap in the childhood literature. We tested a memory model of OR comprehension that explicitly incorporates two working memory (WM) mechanisms, WM storage (WMS) and attention focus switching. Storage was included because it is the one mechanism having been studied extensively in the adult literature and it is becoming a focal point in the childhood literature. Therefore its inclusion represents an 
important extension of this emerging literature. Studying the relation of attention and OR comprehension represents an entirely new research direction. Comprehension of OR sentences by nature involves memory retrieval. Comprehenders must selectively retrieve or reactivate NP1 downstream in the sentence thereby allowing comprehenders to fulfill the movement operation, that is, move NP1 to its proper place in the developing structure. We hypothesize that the ability to switch attention momentarily away from ongoing sentence processing to memory retrieval should be important in supporting comprehenders' reactivation of NP1. We elaborate the potential roles of both mechanisms below. Results of the present study should thus lead to significant new insights into the intersection of WM and OR sentence comprehension in children.

In English, OR structures have occupied a centerpiece in the adult language literature [8-11] and are becoming a focus of interest in the childhood literature $[12,13]$. These structures are of interest because of their unique processing challenge due to their noncanonical nature. The challenge is that NP1 functions grammatically as object and semantically as patient while NP2 functions as subject/agent. It has been argued that comprehenders strive to recover the SVO structure of sentence constituents from noncanonical input [14-16].

Object relatives involve movement of NP1 from the object position to a nonargument position and movement across clausal boundaries. To recover an SVO representation and understand "who did what to whom," a syntactic movement operation is performed to establish what is called a filler-gap dependency, that is, "move" NP1 (filler) to a phonologically empty NP position or trace (gap). To this end NP1 "moves" to its postverb slot, that is, syntactic gap $\left(t_{i}\right)$. To understand the OR sentence "The boy ${ }_{i}$ that the girl had kissed ${ }_{t i}$ on the cheek ran way smiling", NP1 (The boy) must be moved from its fronted object position to its rightful postverb canonical position (the girl had kissed ${ }_{t i}$ the boy). The trace position is linked to the first NP through coindexing $(i)$. It is at this point that NP1 is reactivated at the gap and integrated into the developing syntactic (filler-gap) structure and verb argument structure such that the verb (kissed) can then assign a proper thematic role to each NP, that is, agent to NP2 and patient to NP1.

Online studies of adults' OR comprehension [17-21] and studies with children $[12,13]$ reveal that comprehenders automatically reactivate NP1 (filler) immediately upon encountering the gap. Importantly, it is NP1 that tends to be reactivated, not other NPs (e.g., NP2) appearing before the gap. Such NP reactivation has been demonstrated using a variety of paradigms, including, for example, cross-modal priming $[12,13,17,18]$ and event-related potentials (ERP) [1921].

That memory is an important determinant in complex sentence comprehension has been posited for years [22, 23]. A variety of sentence complexity metrics have been proposed to help explain the nature of the memory load placed on comprehenders by complex grammatical structures. These metrics have included the number of intervening discourse referents [24], number of partially processed dependencies [25], and number of embeddings [23].
Within the memory literature, several models of WM have been proposed. Despite theory dependent differences in architectural and functional details, all models assume WM to be a multimechanism construct whose primary function is to allow individuals to store and maintain information in an active state while simultaneously performing some kind of mental activity [26-30]. Working memory is conventionally measured using some kind of complex span task that includes both a storage and a processing component. In a listening span task, for example, comprehenders are presented sets of sentences and asked to (1) comprehend each sentence, (2) remember a separate item such as a digit or word, and (3) recall as many items in serial order as possible at the end of the set. Item recall is typically the dependent variable. Comprehension is not a dependent variable because individuals typically perform very high on the processing component thereby reducing its variability and usefulness.

In addition to some kind of limited-capacity storage, all models assume a resource-limited attentional mechanism (or central executive) comprising various control mechanisms. It has been argued that these control mechanisms play a critical role in WM performance [26, 27, 30, 31]. One such control mechanism is attention focus switching [26, 32]. The notion of attention switching relates to the idea of bringing items into and out of the focus of attention as an individual performs a WM task. The assumption is that only one attention-demanding step in WM task can occur at any given moment due to an inherent "bottleneck" in the use of central/attentional processes [26]. That is, if one's attentional focus is allocated to processing then it is unavailable for other processes such as storage. Thus, during WM performance, individuals must rapidly switch their attentional (mental) focus between processing and storage such that they allocate attentional focus to the processing activity immediately followed by a momentary switch of attention away from processing to storage to refresh the tobe-recalled items. These rapid switches of attention facilitate overall WM performance by allowing individuals to perform the processing activity with good success while also keeping the storage items active and accessible for later retrieval.

Like adults $[10,24,33,34]$, children also have greater difficulty comprehending OR sentences than canonical sentences (SVO, subject relatives (SR)) [1, 2, 6, 35-37]. To examine children's online grammar processing, Bates and her colleagues $[2,36,37]$ conducted three studies in which 5-17year-old children listened to SVOs, SRs, passives, and ORs. Comprehension was indexed by performance on an agent selection task in which children saw pictures of two animals (agent and patient) on a computer screen while they heard a sentence. They were asked to choose as quickly as possible the animal that performed the action (agent). Findings showed that children's pattern of sentence comprehension mirrored that of adults: accuracy and speed were best for SVOs and SRs, followed by passives and then ORs.

Direct investigations of the association between WM and online OR comprehension in children is very limited $[1,13$, 38]. The few studies that have been conducted have focused exclusively on memory storage. Booth et al. [1], using an individual differences approach, examined the influence of 
simple storage (digit span) and WMS in 8-11-year-old children's comprehension of OR, SR, and SVO sentences. Results showed that (1) simple storage, but not WMS, correlated with OR comprehension and (2) relative to children with higher simple storage, those with less storage were poorer at OR comprehension. However, the lack of association between WMS and OR comprehension may have been due to the nature of the WMS task. Examination of the design of the task suggests that it was too difficult for the children, as indicated by their very low mean scores $(11 \%-23 \%$ correct; variability data were not reported). The absence of correlation between WMS and OR comprehension should thus not be taken as evidence against a potential relation given the possibility that there were problems with the WMS task.

Roberts et al. [13], also using an individual differences approach, examined the relation of WMS and OR sentence processing in children (5-7 year old) and adults. The aim of their study was to examine the relation of WMS and the ability to reactivate NP1 at the gap during OR sentence processing. All of the children and adults completed a conventional WM span task. They also completed a crossmodal picture priming (CMPP) task. The children listened to OR (and filler) sentences in which key NPs corresponding to the agent (the lobster) and patient (the camel) were animals ("Bob fed the camel to which the pink lobster showed his new computer game at his office on Monday morning"). A picture of either the fronted NP (camel) or a control picture (e.g., picture of an object) was presented at one of two locations during the sentence, at the gap position or $500 \mathrm{~ms}$ earlier than gap (control position). Prior to the task, the children were trained to make a classification decision as to whether the prime picture they will see during the sentence is animate or inanimate. They touched the word "Yes" for animal (animate) prime pictures and "No" for object (inanimate) prime pictures, with the assumption being that processing times for the fronted NP (actual patient picture) should be significantly faster than those for control prime pictures at the gap site. The participants also answered a comprehension probe question after each sentence.

Most pertinent to us, the children showed good OR comprehension $(87 \%$ correct). In addition, the children with higher WMS showed faster NP1 reactivation speed than the lower WMS children. Importantly, however, this difference was not statistically tested. Overall, the authors interpreted their results to suggest that, relative to higher WMS children, children with lower WMS may need more time to reactivate and integrate NP1 into a developing structure. This interpretation should be viewed with some caution, as the authors did not statistically test for reactivation speed differences between the high and low WMS children.

To summarize, the childhood literature focusing on the association between WM and OR comprehension is in its infancy. The few studies that have examined this relation have focused exclusively on memory storage, with the results to date inconclusive about its role. And the literature lacks studies investigating the potential role of attention in sentence comprehension. Thus the state of the childhood sentence comprehension literature is that it lacks broader and more integrated memory-based models of comprehension. The present study was designed to begin to address this gap by evaluating a two-mechanism WM model of children's online OR sentence comprehension. The memory mechanisms of interest were WMS and attention focus switching. We investigated online comprehension because we are interested in understanding the influence of memory as comprehension unfolds in the moment. We used a hierarchical linear modeling approach to determine the unique contributions of WMS and attention focus switching. We hypothesized that WMS and attention switching would each account for significant/unique portions of variance in children's OR comprehension. Storage should be important because children need to hold two unintegrated NPs in memory until processing the embedded VP. We also hypothesized that attention switching should be important. We reasoned that children's ability to momentarily switch their attentional focus away from the demands of sentence processing downstream to memory retrieval should facilitate their ability to reactivate NP1 upon encountering the embedded VP.

Because the present study is the first of its kind, we thought it is prudent to begin by using a general modeling approach to address our hypotheses. The advantage of this approach is that it allows us to first establish whether the (1) experimental measures are age sensitive and reliable and (2) WMS and attention focus switching variables each accounts for unique variance in OR comprehension. Positive findings in each instance would provide us with empirical grounds on which we can begin more experimental approaches to model building.

\section{Materials and Method}

2.1. Participants. Forty-four typically developing children 7 through 11 years (7:0-11:11) participated. The mean age of the children was 9.6 years $(\mathrm{M}=116$ months, $\mathrm{SD}=17$ months, and range $=85-144$ months). The sample included 22 boys and 22 girls. All of the children spoke English as their native language. This age range of children was selected because our current research program centering on the intersection of cognitive-linguistic processing focuses on 7-11-year-old children.

Children demonstrated the following: (1) normal-range nonverbal IQ (>85) on the Test of Nonverbal Intelligence3 (TONI-3) [39]; (2) normal-range hearing sensitivity as determined by screening for the frequencies $500 \mathrm{~Hz}, 1 \mathrm{kHz}$, $2 \mathrm{kHz}$, and $4 \mathrm{kHz}$ at $25 \mathrm{~dB} \mathrm{HL}$ [40]; (3) normal or corrected vision; (4) no history of neurological impairment, psychological/emotional disturbance, or attention deficit disorder (as determined by caregiver report); and (5) scores above 1 SD from the mean on the Test for Reception of Grammar2 (TROG-2) [41], a syntax-specific receptive measure that includes sentence structures ranging from simple to complex, and the Peabody Picture Vocabulary Test-3 (PPVT) [42], an index of single word receptive vocabulary knowledge.

2.2. Overview of Experimental Tasks. A modified agent selection task was used to measure children's comprehension of OR sentences. Children listened to sentences and were asked to select the agent of the sentence by selecting one 
of two images appearing at some point during the sentence. This method has been shown to be a reliable and sensitive measure of children's comprehension [2, 36, 37]. Children's WMS was indexed by a conventional verbal WM (listening span) task $[26,27]$ in which the children listened to sets of sentences and were asked to comprehend each and then recall as many sentence-final digits in serial order as they could at the end of the set. Working memory storage was indexed by digit recall, reflecting the ability to store verbal input while simultaneously engaging in sentence comprehension. For this reason, the measure represented a theoretically relevant index of children's WMS for purposes of this study, that is, the ability to store two unintegrated NPs in memory while engaging in ongoing sentence processing. Finally, children's ability to switch attentional focus was measured by an attention switching task in which they listened to series of high and low tones and were asked to maintain an ongoing count of each tone type $[26,32]$. Given that tone presentations switched unpredictably between high and low tones, children needed to switch their focus of attention between the two memory counts to update one of the counts. Because OR comprehension involves the reactivation of NP1 from memory, we hypothesize that NP1 reactivation is supported by the ability to momentarily switch attention away from ongoing sentence processing to memory retrieval. Therefore the present switching task is theoretically relevant to the purposes of the present study and well suited to index children's attentional focus switching ability.

2.3. Stimulus Recording Procedures. The sentence stimuli for the comprehension and WM tasks were read at a normal rate ( 4.4 syllables/s) [43] and with normal prosodic variation by an adult male speaker who had a midwest dialect of the United States. Sentences were digitized $(44 \mathrm{kHz})$, low pass filtered $(20 \mathrm{kHz})$, and normalized for intensity. Sentences were edited to eliminate any noise at the beginning and end of the file.

2.4. Spoken Sentence Comprehension Task. Children's online comprehension of OR sentences was assessed using a modified agent selection task. Children were presented a sentence in combination with two images presented on the touch screen about midsentence, one corresponding to the agent of the sentence and the other to the patient. Children were instructed to touch as quickly as possible the picture of the agent. The task was a hybrid of a conventional agent selection task [2], in which images of the agent and patient are displayed at some point during sentence presentation, and a CMPP task $[12,13]$, in which two images (e.g., image of agent, image of patient) are presented immediately after the embedded verb (syntactic gap) in OR sentences. An agent selection task is designed to assess whether children can identify the agent of the sentence, thereby demonstrating comprehension of "who did what to whom." A CMPP task is designed to determine a child's ability to reactivate NP1 from memory and establish a filler-gap dependency. Sentence understanding is typically assessed indirectly through periodic probe questions following some of the sentences.
In the present study, displaying both agent and patient images midsentence, importantly, allowed us to gather direct evidence of children's online abilities to both (1) create a fillergap dependency and (2) comprehend each sentence, that is, determine "who did what to whom."

2.4.1. Task Design and Stimuli. Thirty experimental OR sentences were created. The sentences were modeled after those used by Love [12]. Across all sentences, NP1 and NP2 were animal names and had a maximum age of acquisition rating of 4.5 years [44-47]. Both NPs were also controlled for imageability $(>500)$, concreteness $(>500)$, and familiarity $(>500)$ on a scale ranging from 100 to 700 [48]. Each embedded verb in the sentence was also familiar to 7-11 -year-old children [49] and controlled for imageability (>450) [44-47, 50]. Pilot testing revealed that 7-8-year-old children labeled and recognized all of the animal pictures with $100 \%$ accuracy. All of the sentences were semantically reversible. Each animal noun appeared as NP1 in half of the sentences and as NP2 in the other half. We selected embedded verbs that were neutral regarding which animal could perform the action on the other. Each animal thus had comparable probability of performing the action on the other thereby minimizing any systematic bias on the part of the children to interpret one NP over another as the agent. This design feature also permitted maximal flexibility in using each animal name in both NP positions.

In keeping with the childhood literature, 30 SR sentences were created as filler items in order to prevent children from potentially developing an input expectancy $[12,13]$. The sentences were constructed following the same constraints as the ORs. All sentences were 12 words in length. The overall acoustic durations of the two sentence types did not differ $\left(\mathrm{OR}_{\text {mean }}=2992 \mathrm{~ms} ; \mathrm{SR}_{\text {mean }}=2951 \mathrm{~ms}\right) F(1,58)=1.60$; $P>$.05. Finally, images of the animals were color drawings standardized for name and image agreement, familiarity, and visual complexity [51].

To obtain a measure of children's online sentence comprehension, the agent and patient images were presented immediately at the offset of the 7th word in both the OR and the SR sentences. In the OR sentences, the 7th word always corresponded to the offset of the embedded verb or gap (The goat that the pig had touched [pic] near the bush was smiling), the earliest point at which children could with certainty assign each NP a thematic role. In the SR fillers, the 7th word always corresponded to the offset of NP2 (The pig that had bumped the sheep ${ }_{\text {[pic] }}$ near the tree was resting). Given the canonical nature of these sentences, it was possible for children to realize that NP1 was the agent immediately upon hearing the embedded VP, before hearing NP2. We elected to display the images after the 7th word in each sentence type for one key control purpose. It ensured that the number of words and temporal duration of input presented in both sentence types would be comparable prior to displaying the images. Relative to sentence onset, no difference occurred between the sentences regarding the temporal onset of the images $\left(\mathrm{OR}_{\text {mean }}=1661 \mathrm{~ms} ; \mathrm{SR}_{\text {mean }}=1623 \mathrm{~ms}\right) F(1,58)=2.36$; $P>.05$. 
2.4.2. Procedure. Children sat in front of a touch screen monitor with their fingers placed in a response ready position on a "+" symbol appearing in the center of the screen. They were told that they would hear a man saying some sentences about animals and that one animal would be doing something to the other. They were told that at some point during the sentence two animal pictures would appear in the middle of the screen. To emphasize the online nature of the task, children were instructed to touch the picture of the animal "that is doing something to the other animal" as quickly as possible. The PC-internal clock started at picture onset. The clock stopped as soon as the child touched a picture. To encourage the children to perform the task in an online manner, they had $5 \mathrm{~s}$ to respond or the trial timed out and would be scored as incorrect. The computer automatically calculated and stored children's response accuracy and time. The task was divided into two blocks and presented in counterbalanced fashion across children. Within each block, no more than two consecutive presentations of any one sentence type occurred. Pilot testing indicated that 7-11-yearold children had no difficulty performing the task.

2.4.3. Dependent Variables. The primary dependent variable was comprehension accuracy (correct agent selection). Children's sentence comprehension speed (agent selection response speed) was also gathered. Comprehension speed allowed us to determine whether the children treated the task as an online or offline measure. Because comprehension regarding "who did what to whom" was being assessed immediately after the 7th word the children did not need to wait until the end of the sentence to respond. Accordingly, we reasoned that responses occurring prior to sentence offset would suggest that the children performed the task in an online manner. By contrast, responses well after sentence offset would suggest that the children waited until after the sentence to respond, treating the task in an offline manner.

2.5. Working Memory Storage Task. Children completed a conventional listening span task to index WMS in the face of processing $[52,53]$. Children were presented sets of SVO sentences and asked to (1) judge the truth value of each sentence, (2) remember a digit presented immediately at sentence offset, and (3) recall as many digits in serial order after the last sentence in the set. Simple SVO sentences were used because WM tasks incorporating simple structures have been shown to be a more robust predictor of sentence comprehension than WM tasks incorporating more complex sentences [54].

2.5.1. Task Design and Stimuli. The task included 60 8-word SVO-type sentences appropriate to the comprehension of 6year-old children $[1,2]$. The primary nouns (NP1, NP2) in the sentences were high familiarity words $[45,49]$. The nouns were controlled for age of acquisition (3.6 years or younger), imageability $(>500)$, concreteness $(>500)$, and familiarity $(>500)$ on scales ranging from 100 to $700[44-47,50]$. The task comprised five sets of sentences, with three trials at each set size. Set sizes were presented in a prescribed random order $(4,3,6,2,5)$ to facilitate children's performance [55].

Half of the sentences required a "Yes" response (sentence had truth value) and half required a "No" response (sentence had no truth value). The "No" sentences involved a semantic violation (e.g., The cat saw the house that was hopping). To ensure the children would process the false items in their entirety prior to making a truth value judgment, the semantic violation occurred in different locations, that is, word position 3, 5, 6, or 8. Following each Yes/No response, a monosyllabic digit (e.g., 1, 2, 3, 4, 5, 6, 8, 9) was presented for later recall. No digit was repeated within a set. This task has proved to be a reliable index of 7-11-year-old children's WMS capacity $(r=.85)$ and to be age sensitive [56].

2.5.2. Procedure. Children sat in front of the touch screen, resting their elbow on a soft pad. They were instructed to place their fingertips of their dominant hand on the "+" sign. They were told that they would hear a man saying some groups of sentences and that they would do three things: (1) comprehend each sentence by responding to its truth value by touching Yes or No (processing episode of the task); (2) remember the digit presented after each sentence; and (3) recall as many digits as possible in the order they were heard. The computer automatically recorded the child's comprehension response accuracy. The child's digit recall was recorded by the examiner on the child's score sheet.

Immediately following a Yes/No response $(100 \mathrm{~ms})$, a digit was automatically presented. Immediately following the digit, the examiner presented the next sentence. Therefore, regardless of any differences across children in the time it took to comprehend the sentence, the immediate presentation of each sentence following the preceding digit was intended to prevent children from rehearsing the digits between sentence trials $[26,56]$. (Great care was taken to ensure that each sentence was presented immediately after the child's response to the previous sentence to minimize opportunity for digit rehearsal $[27,56]$. Because the duration of each processing episode was not fixed as some researchers advocate [26], the WMS task used here should be regarded as self-paced. However, results of previous work have shown similar results (digit recall and comprehension accuracy) using this method $[54,56]$ as controlling the duration of the processing period.) Following the presentation of the entire block of sentences, the monitor turned green, cueing the child to recall the digits.

2.5.3. Dependent Variables. The dependent variable was serial digit recall. The score was based on partial credit-unit scoring on a trial by trial basis, which has been shown to be a more robust index of WMS than absolute span scores [55]. Credit for recall (1 point) was given if the child recalled the digit in serial order and responded correctly to the corresponding sentence. Thus, recall score reflected children's ability to maintain storage in the face of processing. A total recall score of 60 was possible. Percent correct comprehension (correctly judging truth value of the sentences) was also calculated but was not used in the analyses for two reasons. First, item recall is the standard dependent variable in WMS 
tasks [26, 32]. Second, comprehension is usually uniformly high across subjects leading to a lack of variability in scores thus rendering it not very useful.

2.6. Attention Focus Switching Task. The attention focus switching task was one adapted from the adult literature and developed for children by Magimairaj and Montgomery $[56,57]$. The task is based on the Garavan paradigm $[58,59]$ in which participants switch their attentional focus between two different running memory counts as they update and maintain the counts. It is proposed that the memory items are maintained in WM in serial fashion and only one item can occupy the focus of attention at any given moment [58]. The children were presented sets of high tones and low tones and asked to keep count of each tone type that was heard.

2.6.1. Task Design and Stimuli. The stimuli included a high tone $(4 \mathrm{kHz} ; 500 \mathrm{~ms})$ and a low tone $(250 \mathrm{~Hz} ; 500 \mathrm{~ms})$. Each trial consisted of 7-11 tones, with six trials at each sequence length. The task consisted of six blocks of five trials each (30 test trials total). Within a trial, tone stimuli followed a predetermined order. A randomized sequence length was used across trials.

Tone presentations were switch or nonswitch in nature. In nonswitch presentations, the tone presented was the same as the immediately previous one. In switch presentations, the tone presented was different from the previous one. Approximately one-third of the 270 total presentations were switch presentations while the remaining two-thirds were nonswitch [32]. The task has been shown to be both a reliable index of 7-11-year-old children's attention switching and sensitive to age $(r=.83)$ [56].

2.6.2. Procedure. Each child was asked to keep count of how many high tones and low tones he/she heard. The child was asked to be as accurate and fast as possible when verbally updating his/her counts. The children were instructed (and reminded if need be) not to use their fingers to keep count of the memory items, but rather to count in "their head." The task began with a fixation point on the screen for 150, 300 , or $600 \mathrm{~ms}$ (random across trials) followed by a tone [32]. After the child verbally updated the count, he/she pressed the space bar to deliver the next tone. After each tone, the child mentally updated each count (i.e., high tones, low tones), with the time taken by the child to press the space bar to deliver the next tone presumably reflecting the time needed to update each count. For example, if the child already heard two high tones and three low tones and then heard a low tone, he/she would say, "two high, four low." At the end of each trial, the monitor turned green, cuing the child to recall the count totals in any order. The examiner entered the child's count onto the response screen.

2.6.3. Dependent Variable. One dependent variable was selected among several possible [56]-accuracies of attention switching (total correct count) on switch presentations. Switch presentations were selected as the dependent variable because it is these presentations that (1) children need to switch attention on between the memory counts whereas on nonswitch presentations no such switching is required and (2) are of theoretical relevance to the attention switching we hypothesize takes place during OR comprehension. Switch accuracy was also selected because it is the one variable shown to significantly correlate with and predict unique variance in children's WM performance [56] and comprehension of spoken directions [60].

A score of 1 was given for each trial where both counts (high tone, low tone) were updated correctly. If only one of the two counts were correct, the trial would be given a score of .5; if neither count were correct, the trial would be given a score of 0 . The maximum possible score was 30 .

2.7. General Procedures. Children were seen individually for 2-3 sessions, each lasting about 1.5 hours with rest breaks. During the first session, they completed the inclusionary screening and in the remaining sessions they completed the computerized experimental tasks. Prior to testing proper, demonstration and practice items were completed for each task to provide children with familiarity with the tasks. Children clearly understood each task, as evidenced by their performing at or near $100 \%$ accuracy on the practice items, that is, choosing correct agent in the comprehension task, serially recalling digits in the WM task, and providing the correct item counts on the attention switching task. No feedback about performance was provided during practice. Three counterbalanced orders of the tasks were created; approximately equal numbers of children completed each order. Tasks were delivered using E-Prime software [61]. Stimuli were presented binaurally under headphones at a comfortable listening level as determined by the child.

2.8. Data Preparation. For the comprehension task, each child's RT data were trimmed in two phases [62]. In the first phase, upper and lower cut-off criteria were established by visual inspection immediately. All RTs below $500 \mathrm{~ms}$ and above $5000 \mathrm{~ms}$ were removed to prevent extreme RTs from influencing the mean. A small percent of responses was excluded from analysis due to rare interruptions and/or rare failures to respond within $5 \mathrm{~s}$. Overall, less than $5 \%$ of RTs were eliminated. In the second phase, means and standard deviations were computed for each child's RT data set. Outliers were defined as RTs that were \pm 2 SD. Outliers were then removed. Less than $2 \%$ of the RTs were removed.

\section{Results}

3.1. Preliminary Analyses. Diagnostic measures such as histogram of errors, studentized residuals, and Cook's distance were also used to examine cases that might fall far from the regression equation. There were no outliers/influential cases. These findings indicated that the assumptions for the model were satisfied and the conclusions obtained from the model could be endorsed. Collinearity statistics (tolerance and variance inflation factor) indicated that there was no multicollinearity between the predictor variables.) Each experimental task showed good reliability (indexed by 
TABLE 1: Descriptive statistics for all the experimental tasks $(N=$ $44)$.

\begin{tabular}{lcccc}
\hline Experimental tasks & Mean & SD & Range & Reliability \\
\hline $\begin{array}{l}\text { WM storage (percent } \\
\text { digit recall) }\end{array}$ & 70.11 & 16.88 & $35-98$ & .90 \\
$\begin{array}{l}\text { Attention switching } \\
\text { OR comprehension }\end{array}$ & 84.43 & 10.45 & $62-100$ & .78 \\
$\quad$ & & & \\
$\quad$ Accuracy & 73.27 & 22.36 & $17-97$ & .88 \\
$\quad$ Speed & 2568 & 834 & $995-4255$ & \\
\hline
\end{tabular}

Notes. Accuracy scores for working memory storage (WMS), attention switching accuracy, and OR comprehension are expressed as percent correct. Item reliability $=$ Cronbach's $\alpha$ coefficient of reliability.

TABLE 2: Bivariate correlations between the experimental measures $(N=44)$.

\begin{tabular}{lcccc}
\hline Variables & 1 & 2 & 3 & 4 \\
\hline 1 Age & - & $.394^{*}$ & $.614^{* *}$ & $.484^{* *}$ \\
2 WMs & & - & $.440^{*}$ & $.545^{* *}$ \\
3 Attention switching & & .272 & - & $.661^{* *}$ \\
4 OR comprehension & & $.440^{*}$ & $.526^{* *}$ & - \\
\hline
\end{tabular}

Note. ${ }^{* *}$ Significant at $\alpha=.001$ ( 2 tailed); ${ }^{*}$ significant at $\alpha=.01$ (2 tailed). Switching accuracy $=$ attention switching accuracy and OR comprehension $=$ object relative comprehension. Values below the diagonal represent partial correlations with age partialled out.

Cronbach's alpha. Each task also proved to be age sensitive. Table 1 summarizes the descriptive statistics regarding the children's task performances.

The distribution of scores for each of the experimental tasks was evaluated using the Shapiro-Wilk test to determine whether they were normally distributed or not and in need of transformation. The recall accuracy scores for the WMS task were normally distributed $F(44)=.954 ; P=$ .08 . However, attention switching scores were not normally distributed $F(44)=.934 ; P=.02$. Therefore they were converted to square root transformed scores, resulting in a normal distribution (skewness: -.849; kurtosis: -.069). The OR comprehension accuracy scores were also not normally distributed $F(44)=.860: P=.001$ and were converted to square root transformed scores, resulting in a normal distribution (skewness: -.998; kurtosis: -.069). The comprehension RTs were normally distributed $F(44)=.977 ; P=.53$. The SR sentences were not examined nor included in any of the analyses. These sentences were included only as filler items.

Correlation analyses were first run among the experimental measures (transformed and untransformed scores) and age. Results revealed that age significantly correlated with WMS, attention switching, and OR comprehension. In addition, WMS significantly correlated with attention switching. Working memory storage and attention switching also correlated with OR sentence comprehension. Partial correlation analysis, adjusting for age, revealed that WMS and attention switching continued to correlate significantly with OR comprehension. Table 2 displays the results of the correlation analyses.
3.2. Primary Analyses: Predicting Sentence Comprehension. Multiple regression analysis was conducted to determine the total variance in OR sentence comprehension accounted for by age, WMS, and attention switching. The outcome variable was OR comprehension and the predictors were WMS (percent correct recall) and attention switching accuracy (number trials correct). The predictors were entered at once. The analysis used untransformed WMS score and transformed scores for attention switching as well as OR comprehension. Jointly, the age, WMS, and attention switching accounted for $53 \%$ of variance in comprehension $R^{2}=.53$ (adjusted $R^{2}=$ .49) $F(3,40)=14.39 ; P=.0001$.

Hierarchical regression was conducted next to determine the amount of unique variance of each predictor. Age was entered first, followed by WMS in the second step and then attention switching in the third step. Age accounted for $24 \%$ of variance in comprehension. Above age, WMS accounted for an additional significant/unique $15 \%$ variance. Attention switching contributed another significant/unique $14 \%$ variance over and above age and WMS. (Two additional regressions were computed to evaluate the separate influences of nonverbal IQ and receptive syntax knowledge (TROG-2 score) on OR comprehension. The first regression included age, IQ, WMS, and attention switching as the predictors. Results revealed that IQ accounted for no significant additional variance $(<1 \%)$. The second regression included age, TROG-2, WMS, and attention switching. Results revealed no additional significant variance accounted for by TROG-2 $(<1 \%)$.)

Table 3 displays the results of the hierarchical regression analyses.

\section{Discussion}

The aim of this study was to test a two-mechanism memory model of children's online comprehension of OR sentences. The model included WMS and attention focus switching. These mechanisms were selected because of their theoretical relevance to OR comprehension. Storage was hypothesized to be important because comprehenders need to maintain both NP1 and NP2 in memory until encountering the embedded VP later in the sentence, the point at which NP1 should be reactivated to fill the syntactic gap. Storage was also selected because it has only recently begun to be studied as a potential factor in children's OR comprehension. Attention focus switching was hypothesized to be important because we reasoned that the ability to switch attention momentarily away from the current demands of sentence processing to memory retrieval should in part facilitate NP1 retrieval. Also, attention switching was selected because it has yet to be examined in relation to children's OR comprehension and thus its inclusion represents a new and important focal point of investigation.

4.1. Overall Results. The results were clear. First, each experimental measure proved to be age sensitive and to have good reliability. Second, WMS and attention switching correlated with OR comprehension, even after adjusting for age. 
TABLE 3: Hierarchical linear regression analysis for predicting OR sentence comprehension using age, working memory storage (WMs), and attention switching accuracy as the predictors $(N=$ 44).

\begin{tabular}{lcccc}
\hline Variable & $\beta$ & $\Delta F$ & $(\mathrm{df1}, \mathrm{df} 2)$ & $\Delta R^{2}$ \\
\hline $\begin{array}{l}\text { Step 1 } \\
\quad \text { Age }\end{array}$ & .001 & 12.86 & $(1,42)$ & $.234^{* *}$ \\
$\begin{array}{l}\text { Step 2 } \\
\quad \text { Age, WMs }\end{array}$ & .404 & 9.84 & $(1,41)$ & $.148^{*}$ \\
$\begin{array}{l}\text { Step 3 } \\
\quad \text { Age, WMs, } \\
\quad \text { Attention Switching }\end{array}$ & .017 & 11.37 & $(1,40)$ & $.137^{*}$ \\
\hline
\end{tabular}

Note. Total variance accounted for $=53 \% .{ }^{* *}$ Significant at $\alpha=.001$ (2 tailed) and ${ }^{*}$ significant at $\alpha=.01$ ( 2 tailed).

Third, and more importantly, both WMS and attention focus switching predicted children's OR comprehension. Fourth, children appeared to treat the comprehension task as a speeded online measure and did not wait until the end of the sentence to respond, as suggested by the fact that their mean comprehension speed was about 400 ms shorter than the mean duration of the OR sentences. Most critically, that WMS and attention switching were both important to OR comprehension supports our predictions.

4.2. Role of WMS. Children, like adults [8, 9, 63-66], have the capacity and ability to hold both NP1 and NP2 in memory as they comprehend OR sentences. Within the childhood literature, our findings provide stronger and more direct support of the Roberts et al. [13] findings. Recall that these authors implied that WMS is important to children's OR comprehension. The present findings provide stronger support for the idea that children must be able to maintain both NP1 and NP2 in memory until encountering the embedded VP (syntactic gap). Prior to reaching the embedded VP, both NPs remain structurally unintegrated. It is only after processing the VP that the children are able to (1) integrate NP1 into a developing syntactic (filler-gap) and verb argument structure and (2) assign the proper thematic role to each NP, with the agent role being assigned to NP2 and the patient role to NP1. In addition, we assume that NP1 must retain some level of activation while in memory for it to be accessible for reactivation $[63,66]$. The fact that WMS predicted children's comprehension suggests that NP1 did retain some level of activation despite its temporal and linguistic separation from the embedded VP.

It might be argued that the reason why WMS predicted OR comprehension is because both the WMS and comprehension measures were verbal in nature $[67,68]$. On this point, however, the memory literature includes numerous findings of associations between WM and higherorder cognition, irrespective of the modality of the memory and cognitive tasks [69-71]. The present findings would be strengthened if it could be shown that nonverbal WMS tasks or composite verbal and nonverbal WMS tasks also predict children's OR sentence comprehension.
4.3. Role of Attention Focus Switching. Also similar to adults and the emerging childhood literature $[12,13]$, children have the ability to reactivate NP1 from memory and integrate it into a developing structure during OR comprehension. The syntactic movement operation is predicated on the ability to reactivate NP1. We would argue that children's ability to momentarily switch their focus of attention away from the ongoing sentence processing (i.e., processing the embedded VP and following material) to memory retrieval represents a crucial memory mechanism supporting the ability to reactivate NP1. This interpretation is supported by two key findings: (1) attention focus switching accounted for an additional significant portion of unique variance in OR comprehension beyond age and WMS and (2) the children achieved good overall comprehension performance $(73 \%$ correct). We would argue that had the children not been able to switch their attentional focus away from downstream linguistic processing to memory retrieval, they would have misinterpreted the ORs as an SVO, adopting a first noun as agent or order of mention processing strategy. These strategies would have led the children to perform well below chance. That attention switching is critical to children's OR sentence comprehension adds substantively new information to the childhood sentence comprehension literature.

Similar to WMS, some might argue that attention focus switching predicted comprehension because both tasks were verbal. However, this argument is weakened on both theoretical and empirical grounds. Theoretically, controlled attention has been conceptualized by many researchers to be a domain-general construct $[30,69,70]$, cutting across both verbal and nonverbal domains. Empirically, Magimairaj and Montgomery [60] have recently shown that, in addition to simple short-term storage, attention focus switching (a composite index comprising auditory and visual switching tasks) predicts children's ability to comprehend spoken directions. The present study used the same attention switching task as these authors. Though the task was verbally mediated, we would argue that its predictive value lies not in its minimal verbal nature but in its domain-general nature as an index of controlled attention. However, as with the case of WMS, the present findings would be strengthened if it could be shown that nonverbal tasks or composite attention switching tasks also predict OR comprehension.

Others might also argue that attention switching accuracy contributed to the children's OR comprehension because the switching task, too, was just another WMS task in which children needed to maintain two memory counts (high and low tones). However, this argument is significantly weakened given that attention switching accounted for significant and unique variance in comprehension even after accounting for age and WMS. Such findings suggest, then, that attentional switching represents a separable memory mechanism from storage which also helps to support children's OR comprehension.

Finally, some authors [67] might argue for an experiential-based account of why OR sentences are difficult for children. On this view, ORs are difficult not because of their inherent memory challenges per se but because they appear less frequently in the language [72]. On this view, given 
their lower frequency children accrue less experience with this structure than with other structures like SVOs, SRs, and passives. This view, however, does not enjoy strong support from the data either. First, children yielded good overall comprehension $(73 \%$ correct). Such good performance is all the more impressive given that the ORs were devoid of strong semantic plausibility, which rendered agent identification more difficult. One might have expected that this manipulation to have led to especially poor comprehension, but it did not. Second, both WMS and attention switching proved to be significant predictors of OR comprehension.

\section{Conclusions}

The findings from this study add new and important information to the childhood language literature. The extant literature on the potential influence of WMS is very sparse and nonexistent regarding the potential role of attention focus switching on children's OR comprehension. The present findings suggest that WMS and the ability to switch attentional focus away from ongoing linguistic processing to memory retrieval are both important in supporting children's OR sentence comprehension.

The adult sentence comprehension literature has made significant advances in recent years understanding complex sentence comprehension as researchers have developed models of comprehension that include numerous independently motivated memory mechanisms. These advances have also occurred because researchers have employed various experimental approaches to examine the influence of these memory mechanisms on comprehension. The present study used a modeling approach as an initial step to examine the influence of two memory mechanisms on children's OR comprehension. Our findings offer an important empirical base upon which more experimentally-designed studies may begin to be developed. Findings from such investigations should yield important new insights into the intersection of memory and comprehension in children and ultimately more theoretically land empirically motivated models of sentence comprehension.

\section{Conflict of Interests}

The authors declare that there is no conflict of interests regarding the publication of this paper.

\section{Acknowledgments}

The authors would like to thank all of the children and their parents for participating in this study. They also thank Alexander Sergeev for his statistical advice.

\section{References}

[1] J. R. Booth, B. MacWhinney, and Y. Harasaki, "Developmental differences in visual and auditory processing of complex sentences," Child Development, vol. 71, no. 4, pp. 981-1003, 2000.
[2] F. Dick, B. Wulfeck, M. Krupa-Kwiatkowski, and E. Bates, "The development of complex sentence interpretation in typically developing children compared with children with specific language impairments or early unilateral focal lesions," Developmental Science, vol. 7, no. 3, pp. 360-377, 2004.

[3] N. Friedmann, A. Belletti, and L. Rizzi, "Relativized relatives: types of intervention in the acquisition of A-bar dependencies," Lingua, vol. 119, no. 1, pp. 67-88, 2009.

[4] N. Friedmann and R. Novogrodsky, "The acquisition of relative clause comprehension in Hebrew: a study of SLI and normal development," Journal of Child Language, vol. 31, no. 3, pp. 661681, 2004.

[5] B. Kas and Á. Lukács, "Processing relative clauses by Hungarian typically developing children," Language and Cognitive Processes, vol. 27, no. 4, pp. 500-538, 2012.

[6] F. Adani, H. K. J. van der Lely, M. Forgiarini, and M. T. Guasti, "Grammatical feature dissimilarities make relative clauses easier: a comprehension study with Italian children," Lingua, vol. 120, no. 9, pp. 2148-2166, 2010.

[7] F. Arosio, M. T. Guasti, and N. Stucchi, "Disambiguating information and memory resources in children's processing of Italian relative clauses," Journal of Psycholinguistic Research, vol. 40, no. 2, pp. 137-154, 2011.

[8] P. C. Gordon, R. Hendrick, and M. Johnson, "Memory interference during language processing," Journal of Experimental Psychology: Learning Memory and Cognition, vol. 27, no. 6, pp. 1411-1423, 2001.

[9] P. C. Gordon, R. Hendrick, and M. Johnson, "Effects of noun phrase type on sentence complexity," Journal of Memory and Language, vol. 51, no. 1, pp. 97-114, 2004.

[10] J. King and M. A. Just, "Individual differences in syntactic processing: the role of working memory," Journal of Memory and Language, vol. 30, no. 5, pp. 580-602, 1991.

[11] B. McElree, S. Foraker, and L. Dyer, "Memory structures that subserve sentence comprehension," Journal of Memory and Language, vol. 48, no. 1, pp. 67-91, 2003.

[12] T. E. Love, “The processing of non-canonically ordered constituents in long distance dependencies by pre-school children: a real-time investigation," Journal of Psycholinguistic Research, vol. 36, no. 3, pp. 191-206, 2007.

[13] L. Roberts, T. Marinis, C. Felser, and H. Clahsen, "Antecedent priming at trace positions in children's sentence processing," Journal of Psycholinguistic Research, vol. 36, no. 2, pp. 175-188, 2007.

[14] N. Chomsky, The Minimalist Program, MIT Press, Cambridge, Mass, USA, 1995.

[15] N. Hornstein and J. Nunes, "On asymmetries between parasitic gap and across-the-board constructions," Syntax, vol. 5, pp. 26$54,2002$.

[16] R. S. Kayne, The Antisymmetry of Syntax, vol. 25 of Linguistic Inquiry Monograph, MIT Press, Cambridge, Mass, USA, 1994.

[17] J. L. Nicol and M. J. Pickering, "Processing syntactically ambiguous sentences: evidence from semantic priming," Journal of Psycholinguistic Research, vol. 22, no. 2, pp. 207-237, 1993.

[18] D. Swinney, E. Zurif, P. Prather, and T. Love, "Neurological distribution of processing operations underlying language comprehension," Journal of Cognitive Neuroscience, vol. 8, pp. 174$184,1996$.

[19] A. Hestvik, E. Bradley, and C. Bradley, "Working memory effects of gap-predictions in normal adults: an event-related potentials study," Journal of Psycholinguistics, vol. 41, pp. 425438, 2012. 
[20] A. Hestvik, N. Maxfield, R. G. Schwartz, and V. Shafer, "Brain responses to filled gaps," Brain and Language, vol. 100, no. 3, pp. 301-316, 2007.

[21] S. M. Garnsey, M. K. Tanenhaus, and R. M. Chapman, "Evoked potentials and the study of sentence comprehension," Journal of Psycholinguistic Research, vol. 18, no. 1, pp. 51-60, 1989.

[22] J. Kimball, "Seven principles of surface structure parsing in natural language," Cognition, vol. 2, no. 1, pp. 15-47, 1973.

[23] G. A. Miller and S. Isard, "Free recall of self-embedded english sentences," Information and Control, vol. 7, no. 3, pp. 292-303, 1964.

[24] E. Gibson, "Linguistic complexity: locality of syntactic dependencies," Cognition, vol. 68, no. 1, pp. 1-76, 1998.

[25] R. Lewis, "A theory of grammatical but unacceptable embeddings," Journal of Psycholinguistic Research, vol. 25, pp. 93-116, 1996.

[26] P. Barrouillet, N. Gavens, E. Vergauwe, V. Gaillard, and V. Camos, "Working memory span development: a time-based resource-sharing model account," Developmental Psychology, vol. 45 , no. 2, pp. 477-490, 2009.

[27] R. W. Engle, J. E. Laughlin, S. W. Tuholski, and A. R. A. Conway, "Working memory, short-term memory, and general fluid intelligence: a latent-variable approach," Journal of Experimental Psychology: General, vol. 128, no. 3, pp. 309-331, 1999.

[28] A. Baddeley, Human Memory: Theory and Practice, Psychology Press, Hove, UK, 1999.

[29] N. Cowan, Attention and Memory: An Integrated Framework, Oxford University Press, Oxford, UK, 1995.

[30] N. Cowan, E. M. Elliott, S. J. Saults et al., "On the capacity of attention: its estimation and its role in working memory and cognitive aptitudes," Cognitive Psychology, vol. 51, no. 1, pp. 42100, 2005.

[31] M. J. Kane, A. R. A. Conway, M. K. Bleckley, and R. W. Engle, "A controlled-attention view of working-memory capacity," Journal of Experimental Psychology: General, vol. 130, no. 2, pp. 169-183, 2001.

[32] N. Unsworth and R. W. Engle, "Speed and accuracy of accessing information in working memory: an individual differences investigation of focus switching," Journal of Experimental Psychology: Learning Memory and Cognition, vol. 34, no. 3, pp. 616630, 2008.

[33] M. A. Just and P. A. Carpenter, "A capacity theory of comprehension: individual differences in working memory," Psychological Review, vol. 99, no. 1, pp. 122-149, 1992.

[34] M. A. Just, P. A. Carpenter, and T. A. Keller, “The capacity theory of comprehension: new frontiers of evidence and arguments," Psychological Review, vol. 103, pp. 773-780, 1996.

[35] A. Belletti, N. Friedmann, D. Brunato, and L. Rizzi, "Does gender make a difference? Comparing the effect of gender on children's comprehension of relative clauses in Hebrew and Italian," Lingua, vol. 122, pp. 1053-1069, 2012.

[36] R. Leech, J. Aydelott, G. Symons, J. Carnevale, and F. Dick, “The development of sentence interpretation: effects of perceptual, attentional and semantic interference," Developmental Science, vol. 10, no. 6, pp. 794-813, 2007.

[37] E. Von Berger, B. Wulfeck, E. Bates, and N. Fink, "Developmental changes in real-time sentence processing," First Language, vol. 16, pp. 193-222, 1996.

[38] A. R. Weighall and G. T. M. Altmann, "The role of working memory and contextual constraints in children's processing of relative clauses," Journal of Child Language, vol. 38, no. 3, pp. 579-605, 2011.
[39] L. Brown, R. Sherbenou, and S. Johnsen, Test of Nonverbal Intelligence-3, PRO-ED, Austin, Tex, USA, 1997.

[40] American National Standards Institute, Specifications of audiometers (ANSI/ANS-8. 3-1997, R2003), 1997.

[41] D. Bishop, Test for Reception of Grammar-2, The Psychological Corporation, London, UK, 2003.

[42] L. Dunn and L. Dunn, Peabody Picture Vocabulary Test 3, American Guidance Service, Circle Pines, Minn, USA, 2007.

[43] S. E. Weismer and L. J. Hesketh, "The influence of prosodic and gestural cues on novel word acquisition by children with specific language impairment," Journal of Speech and Hearing Research, vol. 36, no. 5, pp. 1013-1025, 1993.

[44] M. Coltheart, “The MRC psycholinguistic database," Quarterly Journal of Experimental Psychology, vol. 33A, pp. 497-505, 1981.

[45] M. J. Cortese and M. M. Khanna, "Age of acquisition ratings for 3,000 monosyllabic words," Behavior Research Methods, vol. 40, no. 3, pp. 791-794, 2008.

[46] L. Fenson, P. S. Dale, J. S. Reznick et al., MacArthur Communicative Development Inventories, vol. 13 of Mental Measurements Yearbook, 2000.

[47] K. Gilhooly and R. H. Logie, "Age of acquisition, imagery, concreteness, familiarity and ambiguity measures for 1944 words," Behavioural Research Methods and Instrumentation, vol. 12, pp. 395-427, 1980.

[48] M. J. Cortese and A. Fugett, "Imageability ratings for 3,000 monosyllabic words," Behavior Research Methods, Instruments, and Computers, vol. 36, no. 3, pp. 384-387, 2004.

[49] A. Moe, C. Hopkins, and R. Rush, The Vocabulary of First-Grade Children, Thomas, Springfield, Ill, USA, 1982.

[50] M. Toglia and W. Battig, Handbook of Semantic Word Norms, Erlbaum, Hillsdale, NJ, USA, 1978.

[51] B. Rossion and G. Pourtois, "Revisiting Snodgrass and Vanderwart's object pictorial set: the role of surface detail in basic-level object recognition," Perception, vol. 33, no. 2, pp. 217-236, 2004.

[52] P. Barrouillet and V. Camos, "Developmental increase in working memory span: resource sharing or temporal decay?" Journal of Memory and Language, vol. 45, no. 1, pp. 1-20, 2001.

[53] D. M. Bayliss, C. Jarrold, D. M. Gunn, A. D. Baddeley, and E. Leigh, "Mapping the developmental constraints on working memory span performance," Developmental Psychology, vol. 41, no. 4, pp. 579-597, 2005.

[54] B. Magimairaj and J. Montgomery, “Children's verbal working memory and the role of processing complexity in predicting spoken sentence comprehension," Journal of Speech, Language and Hearing Research, vol. 55, pp. 669-682, 2012.

[55] A. R. A. Conway, M. J. Kane, M. F. Bunting, D. Z. Hambrick, O. Wilhelm, and R. W. Engle, "Working memory span tasks: a methodological review and user's guide," Psychonomic Bulletin and Review, vol. 12, no. 5, pp. 769-786, 2005.

[56] B. Magimairaj and J. Montgomery, “Children's verbal working memory: relative importance of storage, general processing speed, and domain-general controlled attention," Acta Psychologica, vol. 140, pp. 196-207, 2012.

[57] B. Magimairaj and J. Montgomery, "Examining the relative contribution of memory updating, attention focus switching, and sustained attention to children's verbal working memory span," Child Development Research, vol. 53, pp. 1-12, 2013.

[58] H. Garavan, "Serial attention within working memory," Memory and Cognition, vol. 26, no. 2, pp. 263-276, 1998. 
[59] H. Garavan, T. J. Ross, S.-J. Li, and E. A. Stein, "A parametric manipulation of central executive functioning," Cerebral Cortex, vol. 10, no. 6, pp. 585-592, 2000.

[60] B. Magimairaj and J. Montgomery, "Psycholinguistic mechanisms supporting children's comprehension of spoken directions," (under review).

[61] W. Schneider, A. Eschman, and A. Zuccolott, E-Prime User's Guide, Psychology Software Tools, Pittsburgh, Pa, USA, 2002.

[62] N. P. Friedman and A. Miyake, "The relations among inhibition and interference control functions: a latent variable analysis," Journal of Experimental Psychology, vol. 133, no. 1, pp. 101-135, 2004.

[63] R. L. Lewis, S. Vasishth, and J. A. Van Dyke, "Computational principles of working memory in sentence comprehension," Trends in Cognitive Sciences, vol. 10, no. 10, pp. 447-454, 2006.

[64] J. A. Van Dyke, "Interference effects from grammatically unavailable constituents during sentence processing," Journal of Experimental Psychology: Learning Memory and Cognition, vol. 33, no. 2, pp. 407-430, 2007.

[65] J. A. Van Dyke and B. McElree, "Retrieval interference in sentence comprehension," Journal of Memory and Language, vol. 55, no. 2, pp. 157-166, 2006.

[66] J. A. Van Dyke and B. McElree, "Cue-dependent interference in comprehension," Journal of Memory and Language, vol. 65, no. 3, pp. 247-263, 2011.

[67] T. Farmer, J. Misyak, and M. Christiansen, "Individual differences in sentence processing," in Cambridge Handbook on Psycholinguistics, J. Spivey, M. Joannisse, and K. McRae, Eds., pp. 353-364, Cambridge University Press, Cambridge, UK, 2012.

[68] M. C. MacDonald and M. H. Christiansen, "Reassessing working memory: comment on Just and Carpenter (1992) and Waters and Caplan (1996)," Psychological Review, vol. 109, no. 1, pp. 3554, 2002.

[69] A. R. A. Conway, M. J. Kane, and R. W. Engle, "Working memory capacity and its relation to general intelligence," Trends in Cognitive Sciences, vol. 7, no. 12, pp. 547-552, 2003.

[70] M. J. Kane, D. Z. Hambrick, S. W. Tuholski, O. Wilhelm, T. W. Payne, and R. W. Engle, "The generality of working memory capacity: a latent-variable approach to verbal and visuospatial memory span and reasoning," Journal of Experimental Psychology, vol. 133, no. 2, pp. 189-217, 2004.

[71] H. L. Swanson, "What develops in working memory? A life span perspective," Developmental psychology, vol. 35, no. 4, pp. 9861000, 1999.

[72] G. Fanselow, R. Kliegl, and M. Schlesewsky, "Processing difficulty and principles of grammar," in Constraints on Language: Aging, Grammar, and Memory, S. Kemper and R. Kliegl, Eds., pp. 171-201, Kluwer Academic Publishers, Boston, Mass, USA, 1999. 

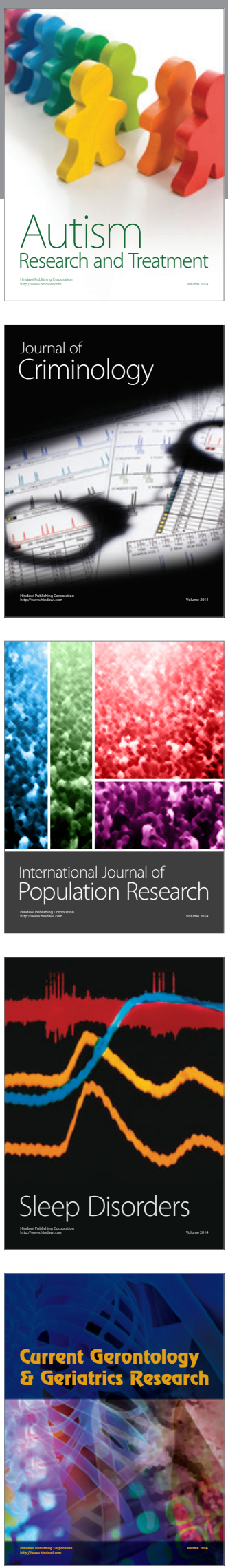
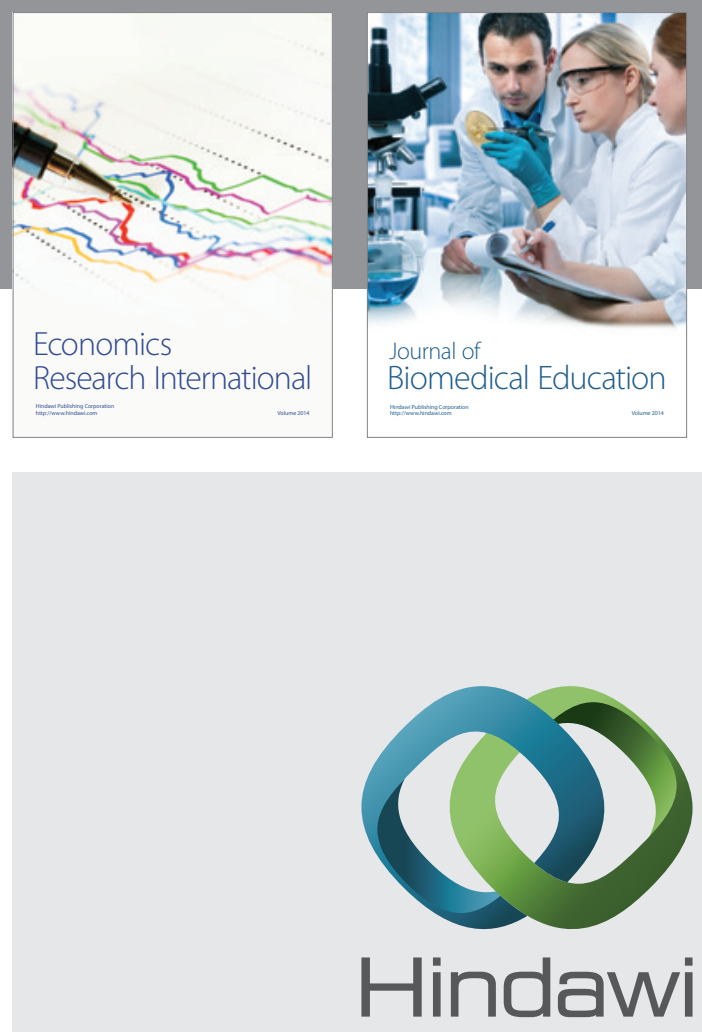

Submit your manuscripts at

http://www.hindawi.com
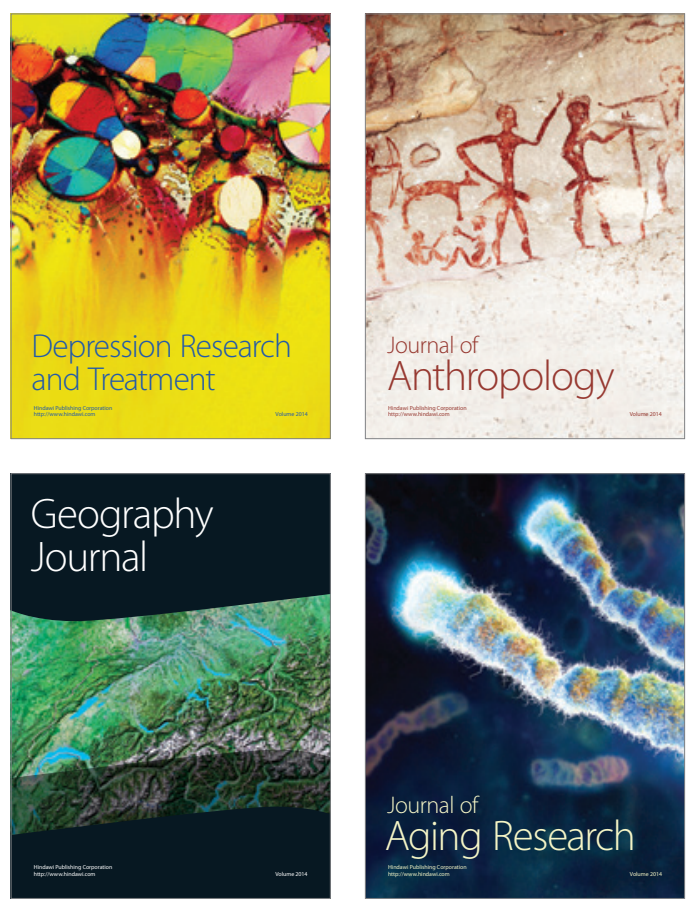
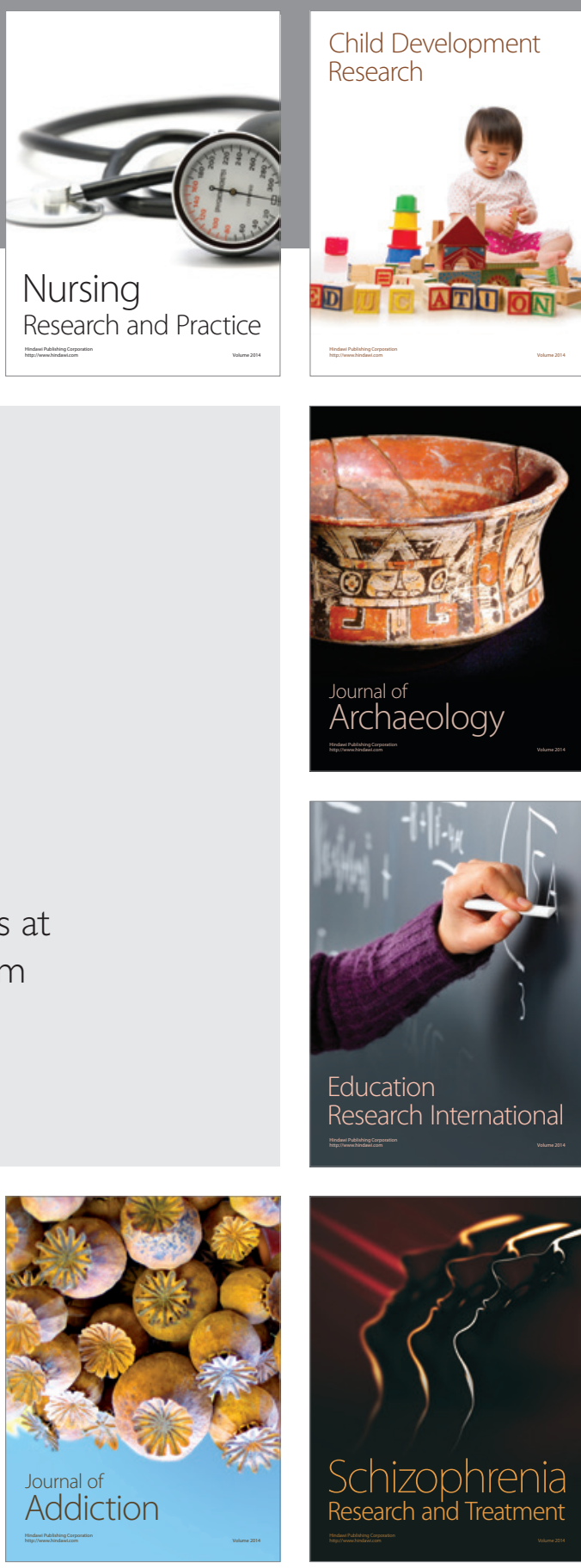

(D)
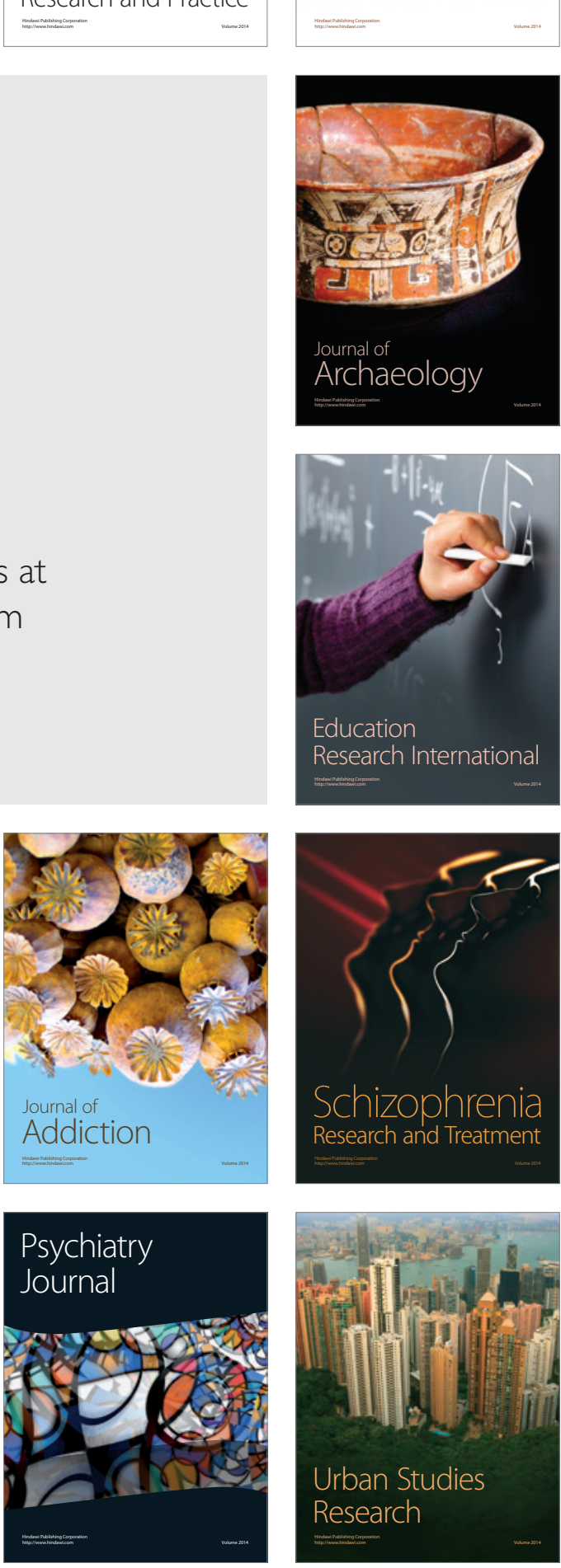